\title{
Combined Microneurosurgical and Endovascular Management of Grade V Occipital Arteriovenous Malformation: Sequential Delayed Embolization Holds the Key!
}

\author{
Ashish Kumar ${ }^{1,2}$ Victor Yang ${ }^{2}$ Leodante Dacosta ${ }^{2}$ \\ ${ }^{1}$ Department of Neurosurgery, Nizam's Institute of Medical Sciences, \\ Hyderabad, India \\ 2 Division of Neurosurgery, Sunnybrook Health Sciences Centre, \\ University of Toronto, Ontario, Canada
}

Indian J Neurosurg 2016;5:47-50.
Address for correspondence Ashish Kumar, MCh, Division of Neurosurgery, Sunnybrook Health Sciences Centre, University of Toronto, 2075 Bayview Avenue, Toronto, ON M4N 3M5, Canada (e-mail: drashishmch@hotmail.com).

A 26-year-old previously healthy woman presented with complaints of sudden-onset headache, decreased level of consciousness, right hemiparesis, and hemianopia. Computed tomography (CT) of the head showed a leftsided parietal hematoma ( - Fig. 1). CT angiography showed a complex arteriovenous malformation (AVM) with a venous varix in the center of the hematoma. Cerebral angiogram revealed a grade V AVM in the left parieto-occipital region measuring $6 \times 4 \mathrm{~cm}$ and supplied by left posterior cerebral artery (PCA) and middle cerebral artery (MCA) and draining into superior sagittal sinus (SSS) and straight sinus via internal occipital vein/vein of Galen (-Fig. 2). Elective sequential endovascular embolization of the nidus was planned over 3 months in four settings. The aim of partial embolization over weeks was to occlude deep feeders and reduce the nidus to allow definitive treatment, either surgical resection or radiosurgery. Gradual flow reduction should also help prevent normal perfusion pressure breakthrough (NPPB) that might happen due to sudden hemodynamic changes during rapid occlusion of large, highflow AVMs.

The treatment plan was developed in a way that each embolization would occlude less than $40 \%$ of the arterial feeders and nidus penetration would be limited. MCA feeders were embolized first, followed by the PCA feeders. Onyx (Covidien; Irvine, California, United States) was used in all sessions. It is composed of EVOH (ethylene vinyl alcohol) copolymer dissolved in DMSO (dimethyl sulfoxide), and suspended micronized tantalum powder to provide contrast for visualization under fluoroscopy. Standard technique was used with Apollo catheters with Mirage guidewires (Covidien; Irvine, California, United States) and Onyx 18

received

April 8, 2015

accepted

November 18, 2015

published online

February 22, 2016 and 34 was injected into the nidus. The choice between 18 and 34 was based on flow characteristics observed during the superselective angiograms. At the end of last session of embolization, only small superficial cortical MCA and PCA feeders were left patent (- Fig. $\mathbf{3}$ ). The little flow lead to the decision to surgically excise the nidus. The patient underwent left-sided occipital craniotomy and excision of the nidus without complications ( - Fig. 4). Postoperative angiogram showed complete resection ( $\mathbf{- F i g . 5}$ ).

Historically, pure surgical treatment for high-grade AVMs is flawed prohibitive morbidity and mortality, and has been largely abandoned. Ujiiye et $\mathrm{al}^{1}$ presented a series of seven patients of grade V AVMs treated with surgery alone. Four of these patients developed hemorrhagic complications either during surgery or postoperatively. However, in recent years this dogma has been challenged with the development of endovascular techniques. Although very large AVM are still challenging, especially in eloquent brain, occlusion was made possible by multimodality therapy, including a combination of embolization, radiosurgery, and/or microsurgical resection. Natraj et al presented their experience in the multimodality management of cerebral vascular malformations spanning 20 years. $^{2}$ Total 265 patients underwent surgery/embolization/radiosurgery or combination of them. Using a multimodality approach, the authors were able to cure $92 \%$ of treated SpetzlerMartin grades I to IV lesions but only $53 \%$ of treated Spetzler-Martin grade V lesions. Increasing age, SpetzlerMartin grade, and rehemorrhage were correlated with a poorer Glasgow Outcome Scale score. van Rooij et al described their initial experience with onyx embolization
DOI http://dx.doi.org/ 10.1055/s-0036-1572368. ISSN 2277-954X. (c) 2016 Neurological Surgeons' Society of India
License terms

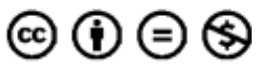




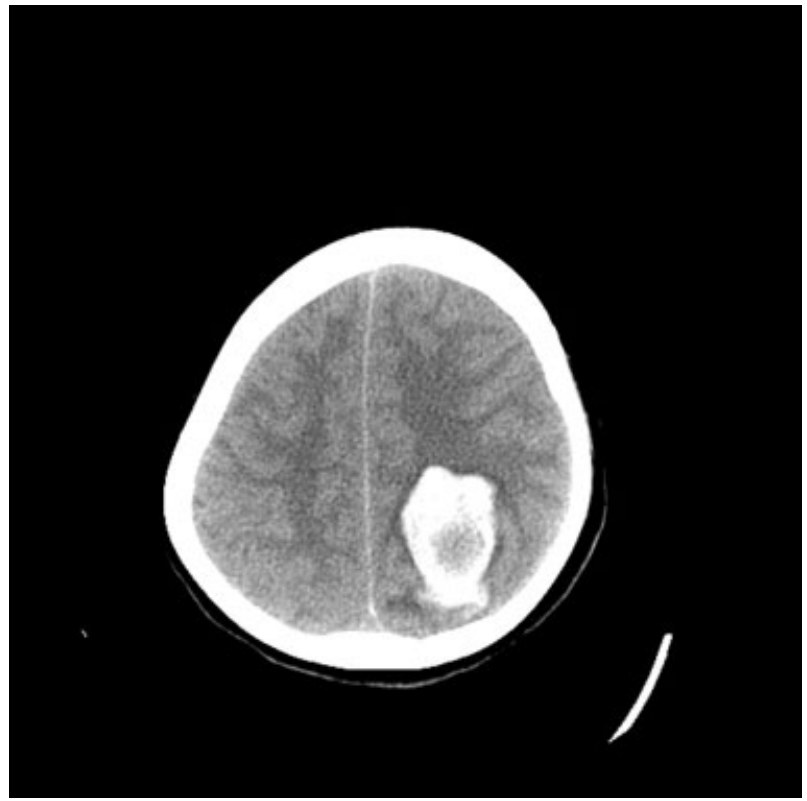

Fig. 1 CT of the head showing left parietal hematoma. where they achieved average reduction in size by $75 \%$. However, complete obliteration was seen in $16 \%$ patients only. $^{3}$ Neurosurgical literature has many references as regards to surgery of high grade AVMs. ${ }^{4}$ However, embolization for these AVMs is not straight forward as it looks. It is important to perform each procedure as a part of a treatment plan, and to avoid the "temptation" of trying to occlude the AVM completely in a single session. This is usually not possible, and the degree of hemodynamic changes induced by "subtotal" embolization can be such that hemorrhagic complications postprocedure are common. To summarize, high-grade AVMs are associated with high morbidity/mortality. However, in selected cases sequential embolization may help reduce their volume facilitating definitive treatment. This report also stresses on the role of delayed surgery after multiple sessions of embolization spaced over months, not days.

In India, where we often find neurosurgical and endovascular disciplines not in perfect harmony, this report would emphasize the need for maintaining perfect balance between them to provide best outcomes for our patient.

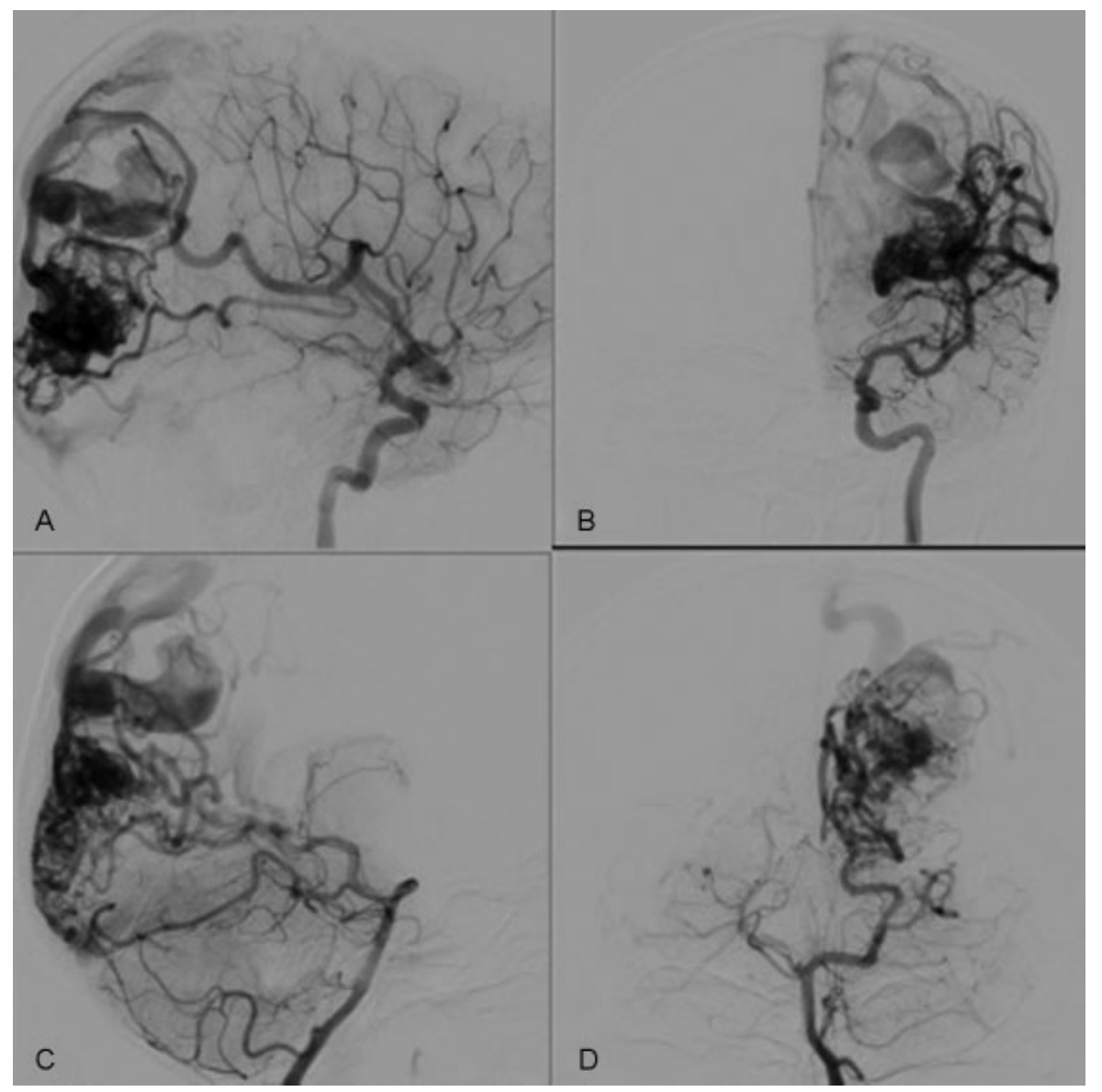

Fig. 2 Digital subtraction angiogram showing grade V AVM with feeders from left MCA (A and B) and PCA (C and D). The nidus is very diffuse and venous varix is seen at the top most likely the cause of hematoma. 


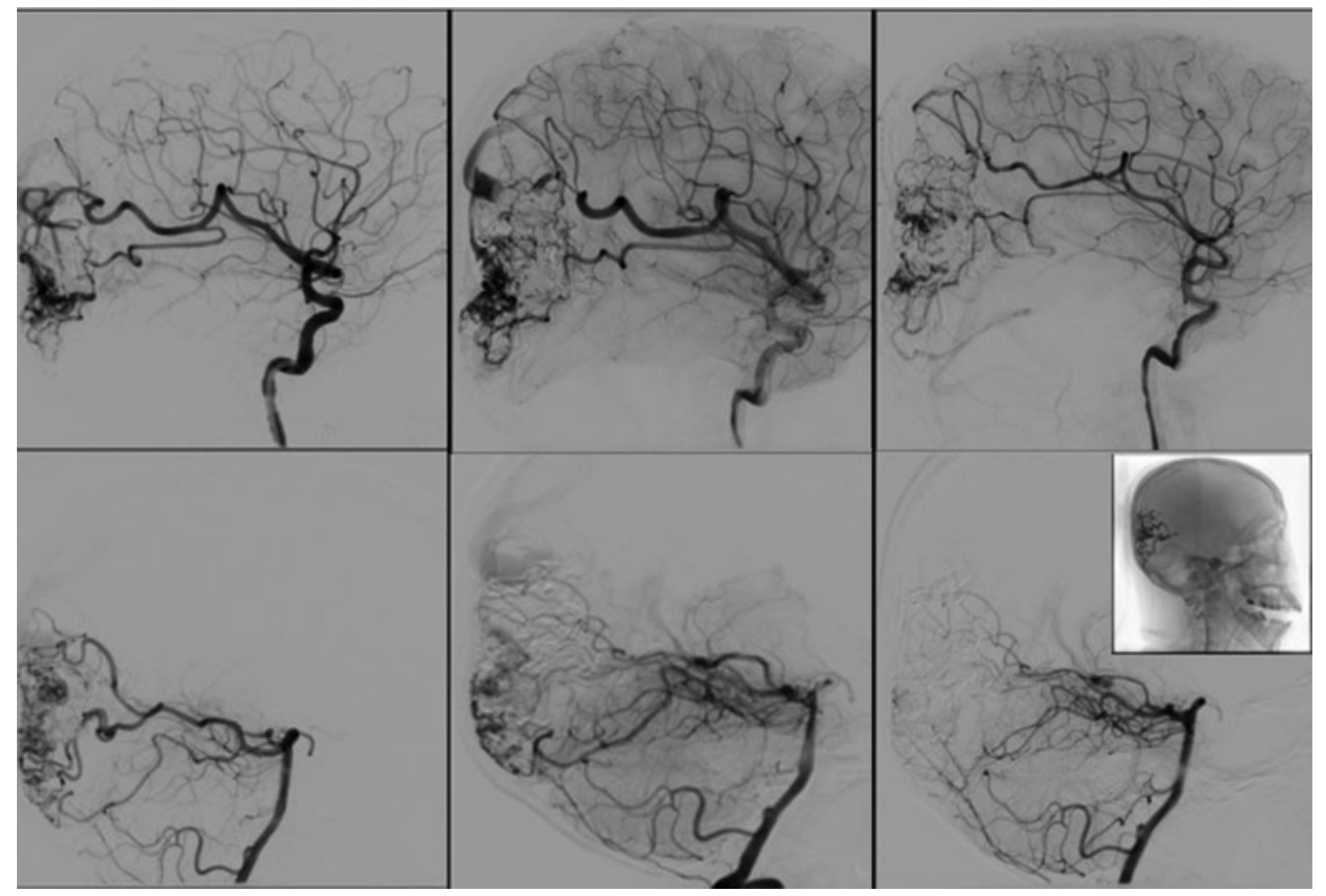

Fig. 3 Sequential obliteration of the nidus in different sessions of embolization (top left to bottom right). Progressive decrease in the MCA and PCA supply is seen. The Onyx cast is also seen in the inset.

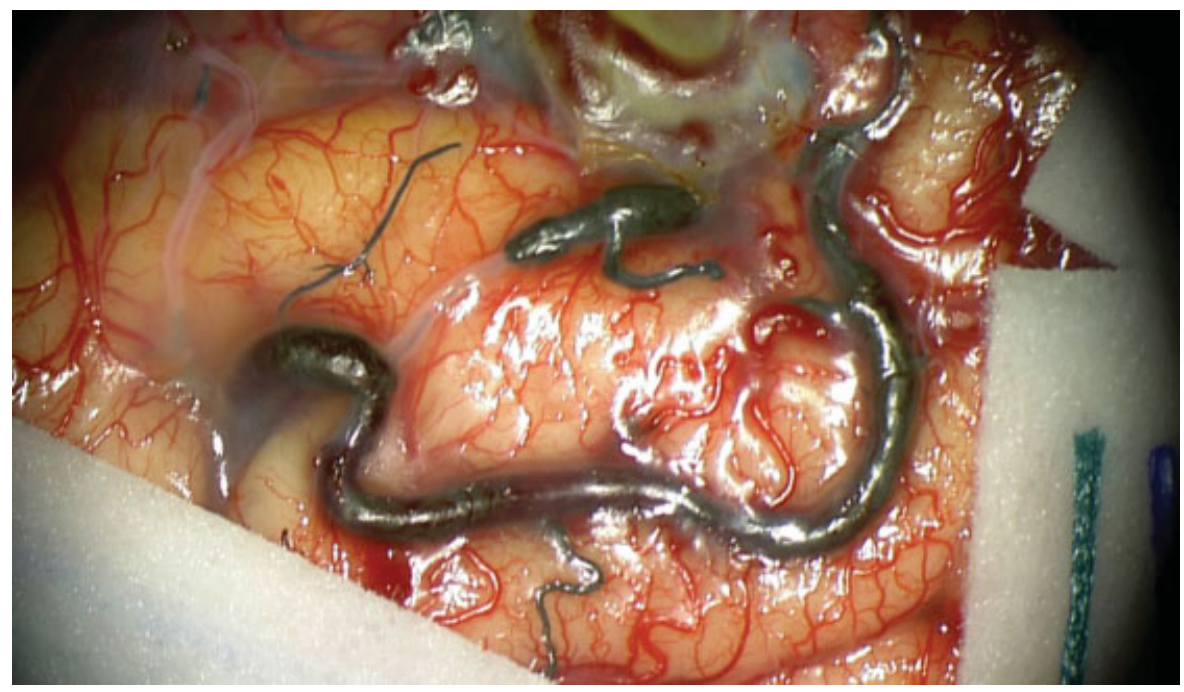

Fig. 4 Intraoperative view after dural opening showing dilated feeders with onyx cast. 
50 Microneurosurgical and Endovascular Management: Occipital AVM Kumar et al.

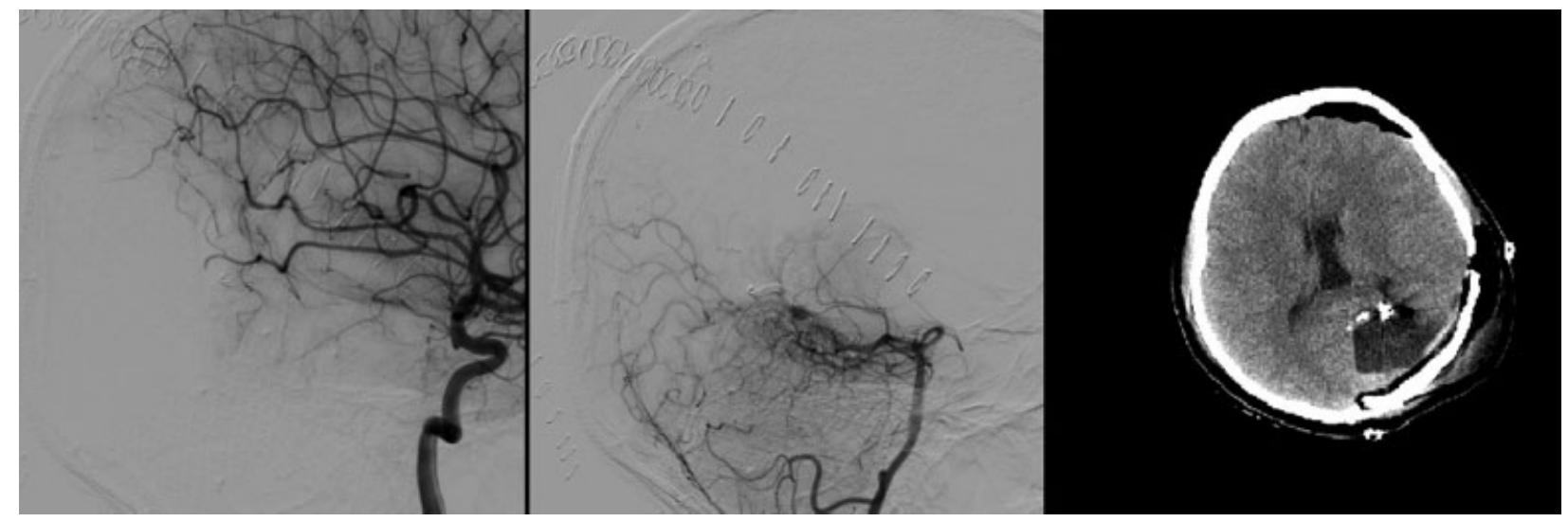

Fig. 5 Postoperative angiogram showing complete resection of the nidus. CT shows residual onyx cast.

\section{References}

1 Ujiie H, Higa T, Hayashi M, Tamano Y, Muragaki Y, Hori T. Surgical management of Spetzler-Martin grade V AVM. J Clin Neurosci 2002;9(Suppl 1):22-25

2 Nataraj A, Mohamed MB, Gholkar A, et al. Multimodality treatment of cerebral arteriovenous malformations. World Neurosurg 2014;82(1-2):149-159
3 van Rooij WJ, Sluzewski M, Beute GN. Brain AVM embolization with Onyx. AJNR Am J Neuroradiol 2007;28(1):172-177, discussion 178

4 Spetzler RF, Martin NA, Carter LP, Flom RA, Raudzens PA, Wilkinson E. Surgical management of large AVM's by staged embolization and operative excision. J Neurosurg 1987;67(1): $17-28$ 